\title{
Terpenoid biotransformations by Mucor species
}

\author{
Eliane de Oliveira Silva • Niege Araçari Jacometti Cardoso Furtado • \\ Josefina Aleu • Isidro González Collado
}

Received: 21 March 2013/Accepted: 22 July 2013/Published online: 3 August 2013

(C) Springer Science+Business Media Dordrecht 2013

\begin{abstract}
Terpenoids are natural products of great interest due to their widespread use in agrochemicals, drugs, fragrances, flavouring and pigments. Biocatalysts are increasingly being used in the search for new derivatives with improved properties especially to obtain structurally novel leads for new drugs which are difficult to obtain using conventional organic chemical methods. This review, covering up to the end of 2012, reports on the application of Mucor species as catalysts in terpenoid biotransformation to obtain new drug targets, enhance pharmacological activity or decrease the unwanted effects of starting material.
\end{abstract}

Keywords Biotransformation · Fungus · Mucor sp. · Terpenoids

E. Oliveira Silva · N. A. J. C. Furtado

Departamento de Ciências Farmacêuticas, Faculdade de Ciências Farmacêuticas, Universidade de São Paulo, Avenida do Café, s/n, Ribeirão Prêto,

São Paulo 14040-903, Brazil

\section{J. Aleu $(\square)$. I. G. Collado ( $\square)$}

Departamento de Química Orgánica, Facultad de Ciencias, Universidad de Cádiz, República Saharaui s/n, Apdo. 40, 11510 Puerto Real, Cádiz, Spain

e-mail: josefina.aleu@uca.es

I. G. Collado

e-mail: isidro.gonzalez@uca.es

\section{Introduction}

Drug discovery using natural products involves the isolation of new molecules from natural products, investigation of their biological activity and the production of more active molecules from them. This latter process that includes chemical modification and microbial transformation is very useful in achieving that. The use of microorganisms that mimic mammalian metabolism to carry out selective transformation reactions make economical and eco-friendly (Green Chemistry principles) microbial transformation advantageous (Kuban et al. 2010).

Microbial biotransformation reactions exhibit high chemo-, regio- and enantioselectivity, producing a wide variety of fine chemicals. They are usually performed using either whole microbial cells or their isolated enzymes. Whole microbial cells, especially those in culture medium, are much less expensive and the enzymes within cells are more stable given that they are in their natural environment and the cofactors are naturally regenerated. Fungi have traditionally been one of the most frequently studied cell systems for biotransformation reactions (Ma et al. 2011).

In 1975, Smith and Rosazza were the first to propose the use of fungi as a model for mammalian metabolism. Metabolites from fermentation using these microorganisms are similar to those released in phase I (functionalization) or less frequently in phase II (conjugation) of xenobiotic modification by mammalian enzymes. Xenobiotic metabolism in mammalian 
systems usually occurs in the liver where hydroxylation, reduction, oxidation, or hydrolysis leads to increased polarity of compounds for better water solubility and eventual excretion. Drugs are also metabolized in the brain where efflux pumps effectively remove them. Therefore, the metabolism ex vivo studies focusing on these organs is particularly interesting (Kutrzeba et al. 2009).

Mucorales is the largest order of the Zygomycetes encompassing important genera such as Absidia, Mucor and Rhizopus. These are mostly fast-growing fungi and are also saprophytic microorganisms. Mycelial elements expand and cover the entire plate in only a few days. The mycelium is described as "cotton candy-like". Asexual spores are borne in sporangia on aerial sporangiophores and in the sexual reproduction the developing sporangiophores become modified to form sexual branches or zygophores. The Mucorales are widespread in nature and can be found on food, grain, vegetables, fruits, nuts and silage (Hollmann et al. 2008).

Dimorphism in Mucorales can be regarded as a differentiation process involving a series of events by which internal stimuli are perceived by cellular sensors and adequately transduced to produce as a response, a morphological change of the cell. As a generalized hypothesis it is proposed that the most important effector which triggers the mycelial-toyeast transition is the establishment of a microaerobic environment. These conditions change the redox potential of the cell where a sensor unchains the morphogenetic response. In Mucor species where microaerobiosis is not sufficient to induce yeast-like growth, it is suggested that the sensor has such a low potential that either additional catabolite repression or an unknown alteration brought about by a high $\mathrm{CO}_{2}$ tension is necessary to initiate the transition (Ruiz-Herrera 1993). It is known that under low levels of glucose or $\mathrm{CO}_{2}$, or both, hyphal development occurred; at high levels, occurs the eliciting yeast development of Mucor sp. (Bartnicki-Garcia 1968).

Some Mucor species are used extensively in biotechnology for enzyme and organic acid production (Hollmann et al. 2008) and are known to have enzyme systems with high proteolytic and lipolytic activity and are capable of producing ethanol and chitosan (Ma et al. 2011). Other species are promised as secondary metabolites producers, such as Mucor rouxianus sp., an endophytic fungus from Taxus chinensis, which was found to produce 10-diacetyl baccatin, baccatin and taxol, being a new candidate for consideration in fermentation technology (Miao et al. 2009).

Considering the great enzymatic potential of Mucor species and the broad range of biological properties of terpenes, this work provides an overview of biotransformation processes successfully applied to the obtention of new derivatives of the most diverse class of secondary metabolites.

\section{Biotransformation of monoterpenoids}

Monoterpenoid compounds are widespread in nature and are often used as fragrances, flavouring or as prototypes of biologically useful substances. They are the subject of many research projects owing to their importance in regulating metabolism in both plants and animals. Biotransformation has been extensively used to carry out stereo- and regioespecific modifications of naturally occurring monoterpenes in order to either enhance their biological activity or to prepare building blocks and enantiomerically pure derivatives that can be used as intermediate molecules in the synthesis of more complex compounds (Souza et al. 2009). For example, in 2009 Esmaeili et al. reported on (-)-menthol biotransformation by Mucor ramannianus obtaining several interesting structures such as sabinane or 1,8-cineole, in high yields (Esmaeili et al. 2009).

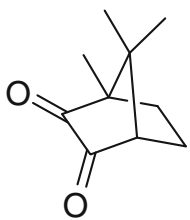

$(-)-1$

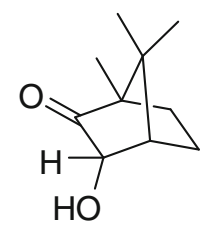

2

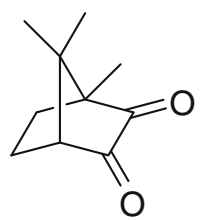

$(+)-1$

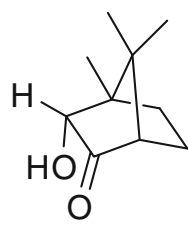

3
Fig. 1 Structures of (-)-(1) and (+)-(1) and reduced derivatives (2) and (3) 
The selective reduction of the monoterpene camphorquinone (1) is an interesting target for biotransformation since in many cases it is possible to achieve the reduction of only one carbonyl group. Mucor is a genus that has proven to be very useful for the selective structural modification of camphorquinonerelated compounds. Therefore, in 1995, Miyazawa et al. reported on the recovery of several keto alcohols from the biotransformation of (-)-(1) and (+)-(1) by five fungal species, including Muсо тисеdo (Miyazawa et al. 1995). In 2009, ten reduced derivatives of $1 R$-(-)-camphorquinone (1) were obtained from biotransformation by Mucor plumbeus. The major derivative produced by $M$. mucedo was $3 \alpha$-hydroxycamphor (2) rather than $2 \alpha$-hidroxycamphor (3), the major product resulting from $M$. plumbeus (Fig. 1). The activity of this fungus was stereoselective with respect to camphorquinone, a feature that can be further exploited for synthetic purposes (Souza et al. 2009).

In 1999, the biotransformation by Mucor piriformis of the monoterpene ketone $(R)-(+)$-pulegone, a potent hepatotoxin, was reported and eight hydroxylated metabolites were obtained. The organism started the transformation either by hydroxylation at the C-5 position or by hydroxylation of the ring methylenes. Although considerable work has been carried out on the mammalian degradation of that monoterpene to menthofuran, considered the toxin responsible for at least $50 \%$ of the toxicity elicited by $(R)-(+)$-pulegone, very little is known about the microbial metabolism of this hepatotoxin. In the study of Madyastha and Thulasiram 1999, the evaluated fungi did not produce the reactive metabolites formed during degradation in the mammalian system. The pathway of transformation of $(S)-(-)$-pulegone by $M$. piriformis was shown to be similar to those of its $(R)-(+)-$ enantiomer. When isopulegone was used as the substrate, the organism isomerized it to pulegone which was then transformed to the same metabolites (Madyastha and Thulasiram 1999).

\section{Biotransformation of sesquiterpenoids}

Sesquiterpenoids are found naturally in plants and insects as semiochemicals, i.e. defensive agents or pheromones, and their biotransformation by different types of filamentous fungi has been extensively studied producing hydroxylated or oxidized products which are difficult to obtain by classical chemical means. Thus, for example, Orabi et al. performed microbial epoxidation of the tricyclic sesquiterpene presilphiperfolane angelato ester using $M$. ramannianus ATCC 9628. The absolute configuration at both oxirane carbons was confirmed by spectral and optical activity data of the hydrolysis product of the novel metabolite $(2 R, 3 R)-(+)-2,3$-epoxyangelic acid (Orabi 2001).

Curcuma aromatica is an important medicinal plant and its rhizome has been used in traditional Chinese medicine as a cancer remedy and to promote bile secretion. Germacrone (4) is one of the major components of this plant and is also the most important and basic sesquiterpene for biosynthesis. Recently, germacrone (4) was reported to exhibit hepatoprotective activity. In an effort to enhance the activity of $\mathbf{4}$, microbial transformation of this compound was performed using Mucor alternata yielding two new compounds, $(1 S, 4 S, 5 S, 10 R)$-isozedoarondiol (5) and $(1 R, 4 S, 5 S, 10 R)$-diepoxy-12-hydroxygermacrone (6) (Fig. 2) (Jia et al. 2008; Niu et al. 2008).

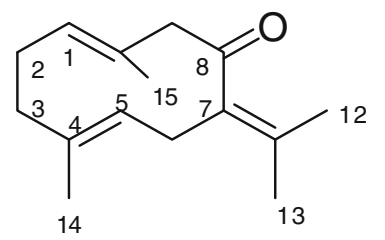

4

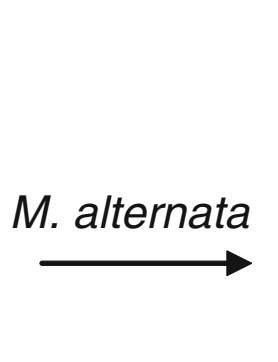

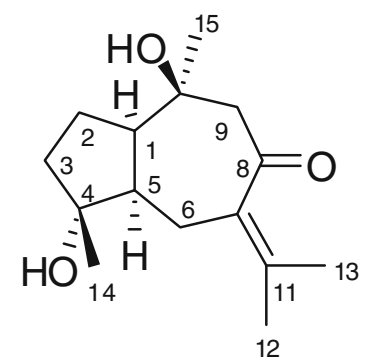

5

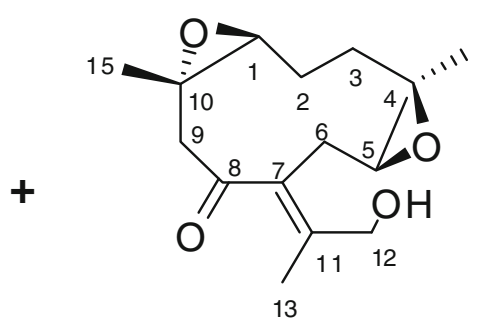

6

Fig. 2 Biotransformation of germacrone (4) by M. alternata 
Sclareolide (7), a natural product isolated from several plant species, displays antifungal, phytotoxic and cytotoxic activities against several human tumor cell lines. Regarding the biotransformation of 7, it has been previously reported that incubation of this compound with $M$. plumbeus afforded 3-ketosclareolide (8), 1 $\beta$-hydroxysclareolide (9) and 3 $\beta$-hydroxysclareolide (10) but when incubated with Mucor miehei it afforded 3-ketosclareolide (8) and 3 $\beta$-hydroxysclareolide (10). Sclareolide (7) and its derivatives were evaluated for in vitro cytotoxic activity against human cancer cell lines following standard procedures. Sclareolide (7) displayed activity against the PC-3 tumor cell line and its derivatives were considered inactive (Cano et al. 2011).

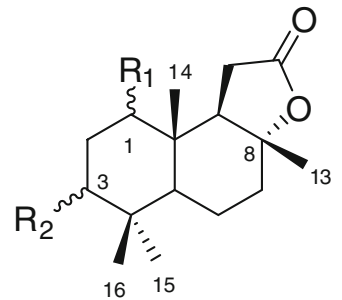

$7 \mathrm{R}_{1}=\mathrm{H} ; \mathrm{R}_{2}=\mathrm{H}$

$9 \mathrm{R}_{1}=\beta \mathrm{OH} ; \mathrm{R}_{2}=\mathrm{H}$

$10 \mathrm{R}_{1}=\mathrm{H} ; \mathrm{R}_{2}=\beta \mathrm{OH}$

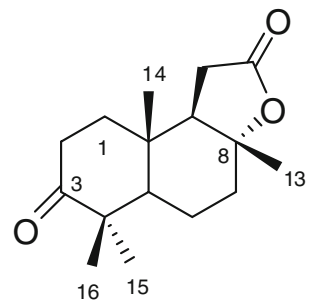

8

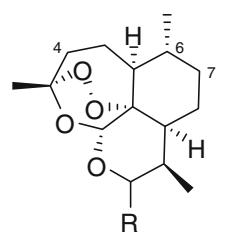

$11 \mathrm{R}=\mathrm{O}$

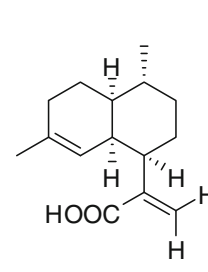

13

$$
12 \mathrm{R}=\mathrm{H}
$$
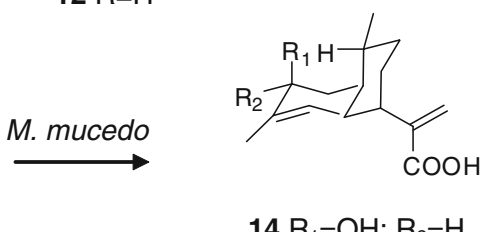

$14 \mathrm{R}_{1}=\mathrm{OH} ; \mathrm{R}_{2}=\mathrm{H}$

$$
15 \mathrm{R}_{1}=\mathrm{H} ; \mathrm{R}_{2}=\mathrm{OH}
$$

Fig. 3 Production of epimeric 3-hydroxyartemisinic acids (14 and 15) from artemisinic acid (13)
Artemisinin (11) is a sesquiterpene lactone isolated from Artemisia annuana L. and an effective antimalaria therapeutic agent. This compound and its derivatives have been used in several Mucor sp. biotransformation studies to predict mammalian drug metabolism. Biotransformation of artemisinin (11) by Mucor polymorphosporus AS 3.3443 (Zhan et al. 2002) and three different strains of M. ramannianus (M. ramannianus 1839, M. ramannianus ATCC 9624 and M. ramannianus ATCC MYA-883) (Parshikov et al. 2005) provided hydroxylated metabolites at C-4, C-6 or C-7. Deoxoartemisinin (12) and artemisinic acid (13) were biotransformed by $M$. ramannianus 1839 (Khalifa et al. 1995; Medeiros et al. 2002) and M. mucedo (Elmarakby et al. 1988), respectively. Epimeric 3-hydroxyartemisinic acids (14 and 15) were obtained from the bioconversion of $\mathbf{1 3}$ (Fig. 3), possible biogenetic precursor of artemisinin (11).

The ability of microorganisms to hydroxylate chemically inaccessible centres is a powerful synthetic tool. In this context, biotransformation of the variety of bridged ring polycyclic sesquiterpenoids provides a useful way of eventually mapping the three-dimensional topology of microbial systems. Fraga et al. explored the hydroxylation of cedrol, 8-epicedrol, $9 \alpha$-hydroxycedrane and $8 \alpha, 9 \alpha$-dihydroxycedrane, compounds in which the position and stereochemistry of a plausible directing hydroxyl group were studied. Hydroxylation of these compounds by M. plumbeus has proven efficient but not stereospecific at the C-3 position (Fraga et al. 1996).

A number of biotransformation studies on sesquiterpenes using $M$. plumbeus have been performed. Thereby, biotransformation by $M$. plumbeus CMI 116688 of valerianol (16), a constituent of valerian oil, and its $1 \alpha$-hydroxy and 1-oxo derivatives, takes place at the $\mathrm{C}-1$ and $\mathrm{C}-10$ positions. The biotransformation of the 1-ketone and $1 \alpha$-alcohol at C-10 involves hydroxylation on the $\beta$-face of the molecule (Arantes et al. 1999).

Sesquiterpenes cadina-4,10(15)-dien-3-one (17) and aromadendr-1(10)-en-9-one (squamulosone) (18) were isolated from the plant Hyptis verticillata which is traditionally used to treat eczema, psoriasis, scabies, athlete's foot, rheumatoid arthritis and a host of coldrelated problems. M. plumbeus ATCC 4740 hydroxylated 17 at C-12 and C-14. However, when the iron content of the medium was reduced, hydroxylation at these positions was also accompanied by epoxidation 
of the exocyclic double bond. The same microorganism converted $\mathbf{1 8}$ to the novel 2a,13-dihydroxy derivative along with four other metabolites (Collins et al. 2002).

Other sesquiterpene compounds such as $\alpha$-santonin (19) and 5 5 -hydroxy-14-eudesm-11-en-3-one (20) have been biotransformed by $M$. plumbeus ATCC 4740. $\alpha$-Santonin (19) was metabolized to 1,2-dihydro$\alpha$-santonin after regiospecific reduction of the carboncarbon double bond on ring A of $\mathbf{1 9}$ (Ata and Nachtigall 2004). Regioselective hydroxylations at C-6 and C-11 were detected for the $5 \alpha$-hydroxy-14-eudesm-11-en-3one (20) biotransformation (Alarcón et al. 2007).

The microbial transformation of (-)-maaliol (21) and (-)-maalioxide (22) by M. plumbeus IMI 116688 produced several hydroxylated metabolites. The biotransformation of 21, which possesses a rigid tricyclic ring system, resulted in the formation of two new hydroxylated products at $\mathrm{C}-1 \beta$ and $\mathrm{C}-9 \beta$ which also contained a carbon-carbon double bond and lacked

Fig. 4 Sesquiterpenoids (16-22) biotransformed by M. plumbeus

the cyclopropane ring of the starting material (Wang et al. 2009). In the case of (-)-maalioxide (22), which possesses a pleasant odour, hydroxylation occurred at $\mathrm{C}-9 \beta, \mathrm{C}-1 \beta$ and $\mathrm{C}-7 \beta$. None of the maalioxide derivatives produced in this study had odours which were noticeably different from that of maalioxide itself (Fig. 4) (Wang et al. 2006).

Partheniol (23) is a fungistatic sesquiterpene which possesses a cyclopropyl ring system and two endocyclic double bonds. Six new partheniol derivative metabolites were isolated from the biotransformation reactions of $\mathbf{2 3}$ with Mucor circinelloides ATCC 15242 (Fig. 5). Several enzymatic systems seem to be involved in these reactions where hydroxylation, hydration, dehydration, carbon-carbon bond cleavage and transannular cyclizations were exhibited (Maatooq 2002).

The cosmetic and fiber industries have been clamoring for efficient production of nootkatone (24), the most important and expensive grapefruit aroma. Biotransformation reactions usually turns out

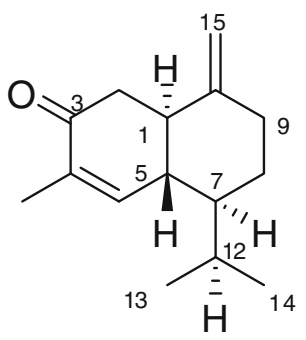

17<smiles>C=C(C)[C@@H]1CC[C@@]2(C)CCC(=O)[C@H](C)[C@H]2C1</smiles>

20

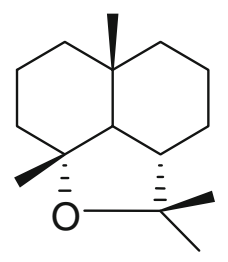

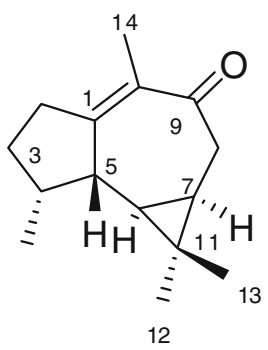

18<smiles>CC1(C)[C@H]2CC[C@@]3(C)CCC[C@@](C)(O)C3[C@H]21</smiles>

21 


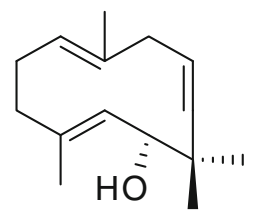<smiles>CC(=O)O[C@H]1C[C@@]2(C)CCC=C(C)C2[C@H]2[C@H]1C2(C)C</smiles>

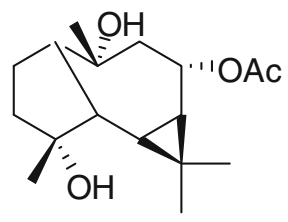<smiles>CC1=CC[C@@H](O)[C@H]2C(C=C(C)CCC1)C2(C)C</smiles>

23<smiles>CC12[C@H]3[C@H](O)[C@@H](O)[C@@](C)(CCC[C@]1(C)O)[C@@H]32</smiles><smiles>C[C@@H]1CCC[C@]2(C)[C@@H]1[C@H]1[C@H]([C@@H](O)[C@@H]2O)C1(C)C</smiles>

Fig. 5 New partheniol (23) derivatives from biotransformation with M. circinelloides ATCC 15242

to be cheaper and more efficient and also provide clean oxidation that does not use any heavy metals, which can be advantageous as a method in the industrial production of nootkatone (24). The commercially available and inexpensive sesquiterpene hydrocarbon valencene (25) from Valencia orange oil can be very efficiently biotransformed into nootkatone (24) using Mucor species (Furusawa et al. 2005).<smiles>[R]C1C=C2CC[C@@H](C(=C)C)C[C@]2(C)[C@H](C)C1</smiles>

\section{$24 \mathrm{R}=\mathrm{H}$}

\section{$25 \mathrm{R}=\mathrm{O}$}

Additionally, the biotransformation by Mucor species of the aristolane-type sesquiterpene hydrocarbon (+)-1(10)-aristolene (26), whose structure resembles that of nootkatone (24), introduced an oxygen function into the cyclohexane ring of aristolene (26), affording the $\alpha, \beta$-unsatured ketone (-)-aristolone (27) which inhibits melanin synthesis (Fig. 6) (Furusawa et al. 2006).

Dehydrocostuslactone (28) and costunolide (29), featuring a guaiane and a germacrane skeletons respectively, are the major active components of the medicinal plant Aucklandia lappa exhibiting anticancer, antioxidant and antifungal properties. With a view to enhancing their solubility and activity, the microbial transformation of $\mathbf{2 8}$ and $\mathbf{2 9}$ by M. polymorphosporus AS 3.3443 was reported, generating stereo- and regio-specific hydroxylation, epoxidation and hydrogenation reactions on the substrates. Several transformed products such as $10 \alpha, 14 \beta$-epoxy-

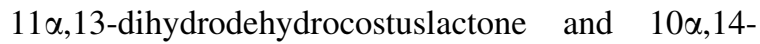
dihydroxy-11 $\alpha, 13$-dihydrodehydrocostuslactone were obtained. These biocatalytic reactions using M. polymorphosporus AS 3.3443 are feasible and powerful routes for directional production of new hydrophilic and bioactive derivatives of $\mathbf{2 8}$ with the advantage of high selectivity, mild reaction conditions and simple operation procedures (Fig. 7) (Ma et al. 2006, 2007).

\section{Biotransformation of diterpenoids}

Fraga et al. spent many years studying diterpenoid (Fig. 8) biotransformations using M. plumbeus, a fungus with low substrate specificity. 17-Acetoxy-kolavenol acetate (30) was chosen as a model substrate to elucidate how the clerodane nucleus is transformed. In this case, M. plumbeus appears to preferentially promote hydroxylation at the C-19 position and to attack the 3,4 double bound. These results indicated that the oxidative 
biochemistry of the microorganism is designed for the A ring in diterpenes. Also, the first step in the biotransformation of $\mathbf{3 0}$ appeared to be deesterification followed by oxidation (Hoffmann and Fraga 1993).

The following studies in microbial transformations of diterpenes with fungi carried out by Fraga et al. included the biotransformation of three manoyl oxide derivatives, jhanol (31), jhanidiol (32) and 1-oxo-jhanol (33), by M. plumbeus CMI 116688. The aim was to obtain substances with functionality similar to that of the bioactive diterpene forskolin (34), which is effective in lowering blood pressure and possesses cardioactive properties (Fig. 8). Biotransformation of $\mathbf{3 1}$ indicated that there was a preference for hydroxylation at $\mathrm{C}-2(\alpha)$ or C-6( $\beta)$ and, to a lesser extent, at C-1( $\alpha)$ or C-11 $(\alpha$ or $\beta)$. The presence of a $1 \beta$-hydroxyl group in 32 inhibited the $6 \beta$ - or the 11-hydroxylation. The epoxidation of the vinyl group was the main reaction followed by hydroxylation at $\mathrm{C}-2 \alpha$ and $\mathrm{C}-3 \beta$. In the case of $\mathbf{3 3}$ there was a preference for $6 \beta$-hydroxylation or epoxidation of the vinyl group. Other hydroxylations were observed at $2 \alpha$, $2 \beta, 3 \alpha, 3 \beta$, or $11 \beta$ positions (Fraga et al. 1998).

In 2001, Fraga et al. aimed to obtain others substances with functionality similar to that of the forskolin (34), the diterpene ribenone (35), isolated from Sideritis canariensis, was also biotransformed by M. plumbeus CMI 116688. Epoxidation of the vinyl group constituted the main reaction and there was a preference for hydroxylation at $\mathrm{C}-1(\alpha), \mathrm{C}-6(\alpha$ or $\beta)$, and $\mathrm{C}-12(\beta)$ and, to a lesser degree, at $\mathrm{C}-7(\alpha)$ and<smiles>C[C@H]1CCC=C2CC[C@H]3[C@@H](C)[C@@]3(C)[C@H]21</smiles>

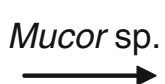

26<smiles>C[C@H]1CCCC2=CC(=O)[C@H]3[C@@H](C)[C@]23C1(C)C</smiles>

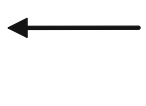

27

Fig. 6 Possible metabolic pathways of $\mathbf{2 6}$ formation by Mucor sp.

C-11( $\beta$ ). Furthermore the reduction of the 3-oxo group to a $3 \beta$-alcohol was observed (Fraga et al. 2001).

Selective functionalization at inactivated carbon atoms has been a difficult challenge in organic

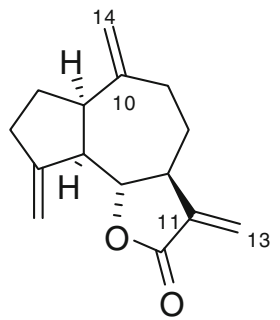

28<smiles></smiles>

29

Fig. 7 Substrates used in biotransformation by $M$. polymorphosporus AS 3.3443<smiles>CC(=O)OC/C=C(/C)CC[C@H]1C2CCC=C(C)[C@@]2(C)CC[C@H]1COC(C)=O</smiles>

30

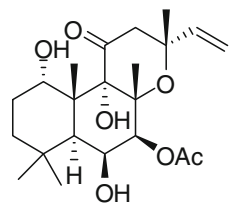

34

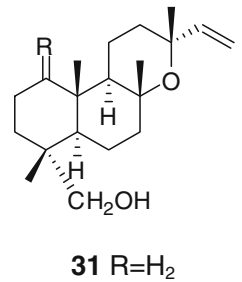

$32 \mathrm{R}=\beta \mathrm{OH}$

$33 \mathrm{R}=\mathrm{O}$

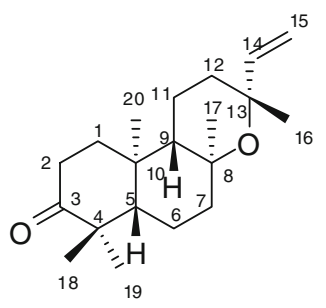

35

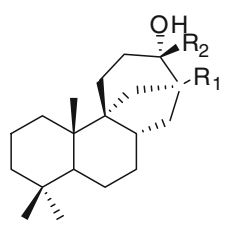

$37 \mathrm{R}_{1}=\mathrm{H} ; \mathrm{R}_{2}=\mathrm{CH}_{2} \mathrm{OH}$

$38 \mathrm{R}_{1}=\mathrm{OH} ; \mathrm{R}_{2}=\mathrm{CH}_{3}$ $39 \mathrm{R}_{1}=\mathrm{OH} ; \mathrm{R}_{2}=\mathrm{H} ; \mathrm{R}_{3}=\beta \mathrm{OH} ; \mathrm{R}_{4}=\mathrm{H}_{2}$ $40 \mathrm{R}_{1}=\mathrm{OH} ; \mathrm{R}_{2}=\mathrm{H} ; \mathrm{R}_{3}=\alpha \mathrm{OH} ; \mathrm{R}_{4}=\mathrm{H}_{2}$ $41 \mathrm{R}_{1}=\mathrm{OH} ; \mathrm{R}_{2}=\mathrm{H} ; \mathrm{R}_{3}=\mathrm{H}_{2} ; \mathrm{R}_{4}=\mathrm{OH}$ $42 \mathrm{R}_{1}=\mathrm{H} ; \mathrm{R}_{2}=\mathrm{OH} ; \mathrm{R}_{3}=\mathrm{H}_{2} ; \mathrm{R}_{4}=\mathrm{OH}$

Fig. 8 Diterpenoids biotransformed by M. plumbeus 
synthesis using chemical methods and in this way, microbiological methods have frequently been used for this purpose. Stemodane diterpenoids are attractive because of their structural similarity to aphidicolin (36), an antiviral agent and a specific inhibitor of DNA polymerase. Thus, two stemodane derivatives,

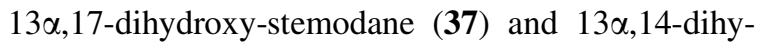
droxy-stemodane (38), were biotransformed by $M$. plumbeus. The main hydroxylations occurred on ring A, especially at C-2( $\alpha)$ and C-3( $\beta)$. Fraga et al. concluded that the $\mathrm{C}-3$ position (equatorial) is the most frequently hydroxylated by $M$. plumbeus in diterpenoids and does not depend on the absolute configuration of the substrate. A competitive hydroxylation at the near axial methyl at C-4, probably produced by the same enzyme, was also obtained in the incubations carried out with this fungus. Hydroxylation of the axial or equatorial methyl at C-4 was also observed for other diterpenes. Positions C-2 or C-3 were also frequently oxidized by this fungus to an oxo group as occurs with manoyl and manoyl oxide derivatives at C-2 and with stemodane derivatives at C-3 (Fraga et al. 2004).

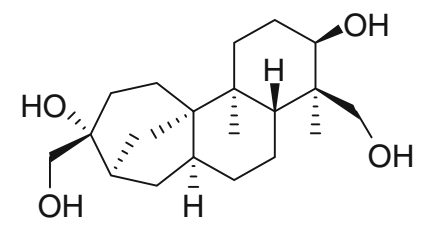

36

On the other hand, biotransformation reactions using M. plumbeus CMI 116688 of two ent-kaurene derivatives, epicandicandiol (39) and candicandiol (40), which are epimeric at C-7, were also studied by Fraga et al. A spatial change in the orientation of the hydroxyl group at C-7 from axial in $\mathbf{3 9}$ to equatorial in $\mathbf{4 0}$ affected the way these kaurenes bind to the oxidative enzymes affording a different hydroxylation pattern in the $\mathrm{A}$ and $\mathrm{B}$ rings (Fig. 8). Thus, the main difference between the two feedings was the formation of a $3 \alpha$-hydroxylated derivative of $\mathbf{3 9}$ in the first and a $9 \beta$-hydroxylated derivative of $\mathbf{4 0}$ in the second (Fraga et al. 2003).

Continuing with their studies aimed to develop models to explain the hydroxylation of diterpenes by M. plumbeus, in Fraga et al. (2010) described the results of the microbial transformation of two entkaurene derivatives, $15 \alpha, 18$-dihydroxy-ent-kaur-16ene (41) and 15 $\alpha, 19$-dihydroxy-ent-kaur-16-ene (42), which are epimeric at C-4. Hydroxylations at C-3( $\alpha)$,
C- $6(\alpha), C-9(\beta)$ or $C-11(\beta)$ and epoxidation of the exocyclic double bond occurred in both fermentations. This means that a change in the spatial orientation of the hydroxymethylene group at C-4, from equatorial in $\mathbf{4 1}$ to axial in $\mathbf{4 2}$ does not affect the way in which these ent-kaurenes are recognized by the enzyme involved in these reactions. Additionally, $O$-glucosides were formed at C-19 in the feeding of $\mathbf{4 2}$ but not of 41, this being the first time that ent-kaurene derivatives of this type were formed in a biotransformation by a Mucor species (Fraga et al. 2010).

In several diterpene studies Fraga et al. obtained entkaur-11-ene derivatives, together with other diterpenes, from species of the genus Sideritis, endemic to the Canary Islands. These ent-kaur-11-ene diterpenes are rare in nature, having been isolated only from plants containing ent-trachylobane diterpenes. Fraga et al. have proposed that diterpenes with an ent-trachylobane skeleton may be the biogenetic precursors of ent-kaur11-ene derivatives. Consequently, they decided to attempt the biotransformation of trachinodiol (43) by M. plumbeus with the aim of obtaining the corresponding ent-kaur-11-ene derivative sicanatriol (44) which is difficult to prepare using chemical methods (Fig. 9). This biotransformation must occur by enzymatic abstraction of an $\mathrm{H}-11$, in a way similar to that postulated by this group in plants of the Sideritis genus where ent-trachylobane and ent-kaur-11-ene derivative diterpenes coexist. The functionalization of $\mathrm{C}-11$ in $\mathbf{4 3}$ to form $\mathbf{4 4}$ was likely favored because this carbon is allylic to the cyclopropane ring. This is analogous to the functionalization of $\mathrm{C}-15$, allylic to the 16,17 double bond in ent-kaur-16-ene diterpenes, which was observed by Fraga et al. (2003) in the biotransformation of candicandiol (37) and epicandicandiol (36) using $M$. plumbeus. Therefore, the same enzyme may be responsible for both bioreactions (Fraga et al. 2011).

M. plumbeus was also used in diterpenoid biotransformation studies by several other researchers (Hanson et al. 2003; García-Granados et al. 2007). Thus, in order to obtain homochiral intermediates for the hemisynthesis of biologically relevant molecules, Aranda et al. carried out a study of the biotransformation of labdane-derived terpenoids. Microbial transformation of five labd-14-ene derivatives (Fig. 10) was performed with M. plumbeus ATCC 4740. Sclareol (45) quantitatively afforded a mixture of triols and the incubation of other labdane derivatives, including manool (46), the corresponding $7 \alpha$-hydroxy derivative 
Fig. 9 Biotransformation of diterpenes with an enttrachylobane (43) skeleton to ent-kaur-11-ene (44) derivative by $M$. plumbeus

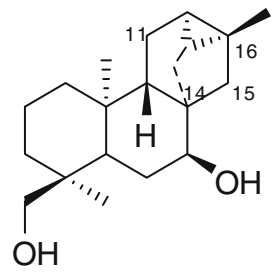

43

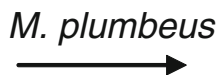

44
(47), manoyl oxide (48) and sclareolide (49) resulted in a mixture of several new hydroxylated and ketoderivatives. The results from this study showed that M. plumbeus may be considered a versatile microbial tool for the regio- and stereoselective introduction of hydroxyl groups into cyclic diterpenes of the labdane family. In addition to the $3 \beta$-position, highly favoured in 45 , the $1 \beta-, 2 \alpha-, 6 \alpha-, 7 \alpha-, 18$ - and 19-positions were also hydroxylated. It could be that the presence of an additional exocyclic double bond at $\mathbf{4 6}$, or a lactone structure at $\mathbf{4 9}$, or an ether bridge at $\mathbf{4 8}$, dramatically enhanced the occurrence of degradative reactions in the microorganism used (Aranda et al. 1991).

A great variety of ent-kaurenoid tetracyclic diterpenoids have been isolated from Chinese medicinal plants. Many of these compounds exhibit tumorinhibition properties. This prompted Boaventura et al. to make compounds of this type by means of diterpenoid biotransformations. Whilst the introduction of the 15-ketone adjacent to a 16-ene is relatively easy by chemical means, the introduction of more remote functionality may be better performed by microbiological means. Thus, this group described the biotransformation of methyl ent-15-oxokaur-16-en19-oate (50) by M. plumbeus ATCC 4740, obtaining the single metabolite (51) (Boaventura et al. 1995).

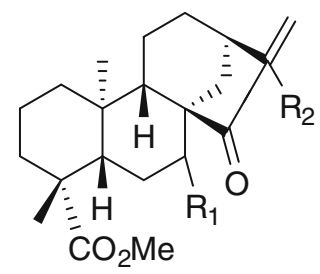

$$
\begin{aligned}
& 50 \mathrm{R}_{1}=\mathrm{R}_{2}=\mathrm{H} \\
& 51 \mathrm{R}_{1}=\mathrm{R}_{2}=\mathrm{OH}
\end{aligned}
$$

Three diterpenes with stemodane and stemarane skeleta, stemodin (52), stemodinone (53) and stemarin (54), which possessed mild antiviral and cytotoxic properties, were incubated with $M$. plumbeus ATCC 4740 as part of a programme to produce analogues of biologically active natural products using fungi. Hydroxylated metabolites were obtained at the C-2 $\alpha$, $\mathrm{C}-3 \beta, \mathrm{C}-6 \beta, \mathrm{C}-11 \beta, 13$ and 14 positions from 52, at C- $6 \alpha, C-12 \alpha$ and C-13 from 53 and at C- $2 \alpha, C-8, C-13$ and C-19 from 54 (Chen et al. 2005).

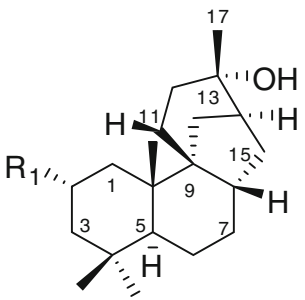

$52 \mathrm{R}_{1}=\mathrm{OH}$

$53 \mathrm{R}_{1}=\mathrm{O}$

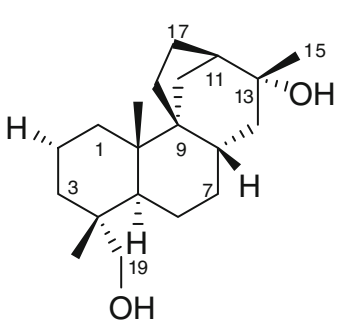

54

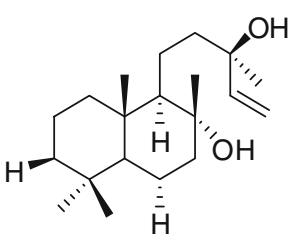

45

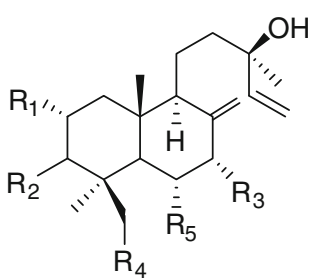

$46 \mathrm{R}_{1}=\mathrm{R}_{2}=\mathrm{R}_{3}=\mathrm{R}_{4}=\mathrm{R}_{5}=\mathrm{H}$

$47 \mathrm{R}_{1}=\mathrm{R}_{4}=\mathrm{R}_{5}=\mathrm{H} \mathrm{R}_{2}=\mathrm{R}_{3}=\mathrm{OH}$

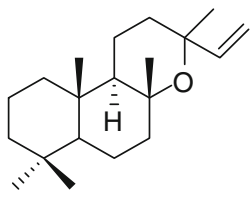

48

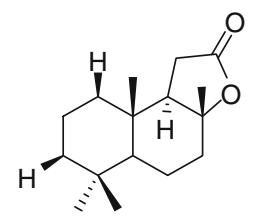

49
Fig. 10 Labd-14-ene derivatives used in the biotransformation by $M$. plumbeus ATCC 4740 by Aranda et al. 
Some mulinane-type diterpenoids such as mulin11,13-dien-20-oic acid (55), have been shown to display trichomonicidal effects and to have trypanocidal activity as well. In this connection, Areche et al. reported the biotransformation of $\mathbf{5 5}$ by M. plumbeus and two new mulinane-type derivatives (56 and 57) were obtained (Areche et al. 2008).

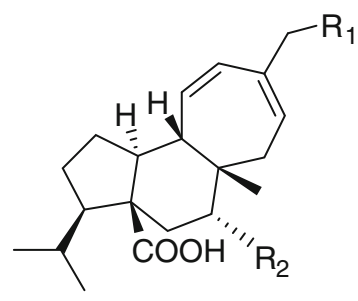

$$
\begin{aligned}
& 55 \mathrm{R}_{1}=\mathrm{OH} ; \mathrm{R}_{2}=\mathrm{H} \\
& 56 \mathrm{R}_{1}=\mathrm{H} ; \mathrm{R}_{2}=\mathrm{H} \\
& 57 \mathrm{R}_{1}=\mathrm{OH} ; \mathrm{R}_{2}=\mathrm{OH}
\end{aligned}
$$

Many Mucor species other than M. plumbeus have been used in the taxoid-type diterpenoid biotransformation. As taxoids are a series of complex natural compounds with potential anti-cancer bioactivity, their structural modification by fungi from the yew tree may be of some value. 10-Deacetyl-7-epitaxol (58), a taxoid that is more abundant than taxol in the bark of Taxus yunnanensis, was biotransformed by Mucor sp. isolated from the inner bark of the same yew species. As result, three metabolites $(\mathbf{5 9}, \mathbf{6 0}$ and $\mathbf{6 1})$ were obtained (Zhang et al. 1998).
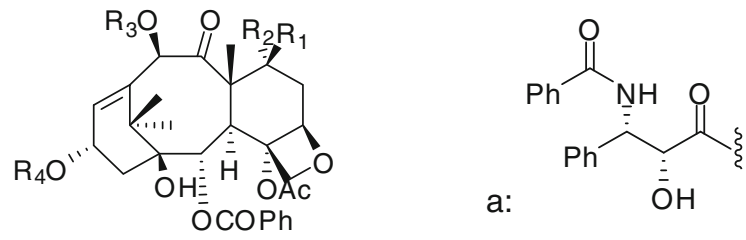

$$
\begin{aligned}
& 58 \mathrm{R}_{1}=\mathrm{H} ; \mathrm{R}_{2}=\mathrm{OH} ; \mathrm{R}_{3}=\mathrm{H} ; \mathrm{R}_{4}=\mathrm{a} \\
& 59 \mathrm{R}_{1}=\mathrm{H} ; \mathrm{R}_{2}=\mathrm{OH} ; \mathrm{R}_{3}=\mathrm{H} ; \mathrm{R}_{4}=\mathrm{H} \\
& 60 \mathrm{R}_{1}=\mathrm{OH} ; \mathrm{R}_{2}=\mathrm{H} ; \mathrm{R}_{3}=\mathrm{H} ; \mathrm{R}_{4}=\mathrm{a} \\
& 61 \mathrm{R}_{1}=\mathrm{OH} ; \mathrm{R}_{2}=\mathrm{H} ; \mathrm{R}_{3}=\mathrm{H} ; \mathrm{R}_{4}=\mathrm{H}
\end{aligned}
$$

Cancer treatment with chemotherapeutic drugs is frequently hindered by intrinsic or acquired resistance of tumor cells. In both cases, tumors can become resistant to a variety of antineoplastic drugs of diverse structures and mechanisms of action, a process termed multi-drug resistance (MDR). Many natural taxanes and chemical derivatives with MDR-reversal activity have been reported and it is very interesting to investigate the MDR-reversal activity of these compounds. The structural diversification of several 4(20),11(12)-taxadienes, including sinenxan A (62) by Mucor genevensis JCM 10585 was achieved. Reactions were diverse and included hydroxylation, epoxidation, oxidation, hydrolysis, acylation, $O$-alkylation, $O$-glycosylation, rearrangement, etc. Furthermore, one chemical derivative, $9 \alpha$-cinnamoyl sinenxan A (64) from enzymatic product $9 \alpha$-hydroxyl sinenxan A (63) (Fig. 11), displayed significant reversal activity toward three MDR tumor cell lines (Dai et al. 2008).

Microbial conversion of $\mathbf{6 2}$ by M. genevensis JCM 10585 was performed to diversify taxane sinenxan A (62) for direct evaluation of MDR reversal activity and for further chemical derivatization therein, and the MDR reversal activities of the three major products towards paclitaxel-resistant human non-small cell lung cancer (NSCLC), were evaluated. In conclusion, sinenxan A (62), a readily available taxoid from Taxus cell cultures, was specifically methylated and epoxidized by the fungus $M$. genevensis JCM 10585 . Bioassays showed that product $\mathbf{6 5}$ was more active than verapamil against taxol-resistant human NSCLC cells (Yang et al. 2007a).

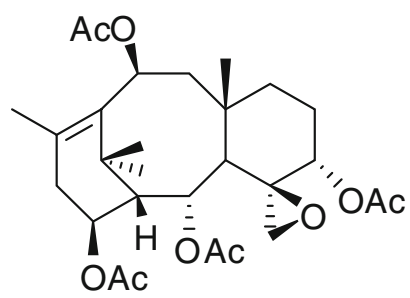

65

Stevioside (66) is a noncaloric sugar substitute used in Japan and Brazil. It is able to regulate blood glucose levels by enhancing not only insulin secretion but also insulin utilization in insulin-deficient rats. Steviol (67), an aglycone of stevioside (66), correspond to the major compound formed during hydrolysis of $\mathbf{6 6}$ by intestinal microflora from various animal species, including man. The use of selected microorganisms as models for "xenobiotic metabolism" has been successfully exploited in mammalian metabolism studies 


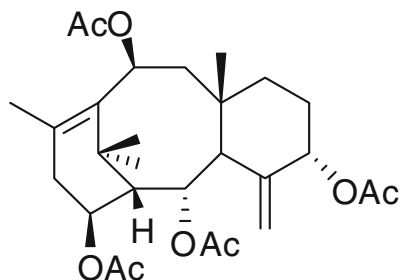

62

\section{M. genevensis}

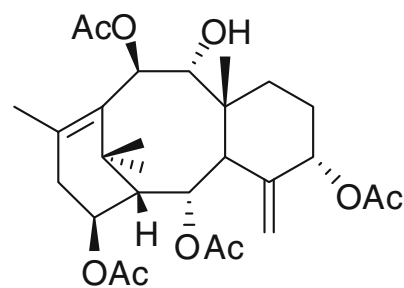

63

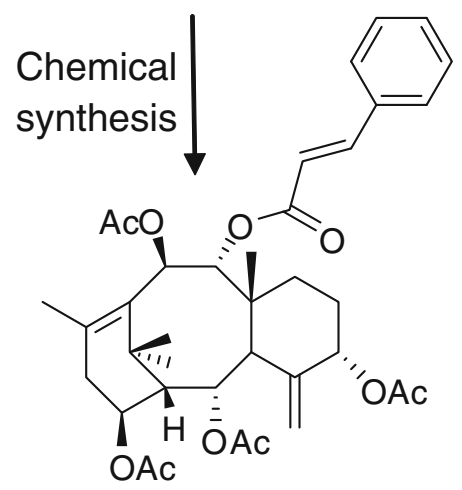

64
Fig. 11 Preparation of $9 \alpha$-cinnamoyl sinenxan A (64)

for many classes of biologically active natural and synthetic compounds. The bioconversion of $\mathbf{6 7}$ by Mucor recurvatus MR36 was undertaken by Yang et al. as part of a program to produce mammalian metabolites and/or novel analogs for biological assays. In addition, a GRE (glucocorticoid response element)mediated luciferase reporter assay was also used to initially screen the derivatives 68,69 and 70 , obtained from 67, which displayed a significant effect as glucocorticoid agonists (Yang et al. 2007b).

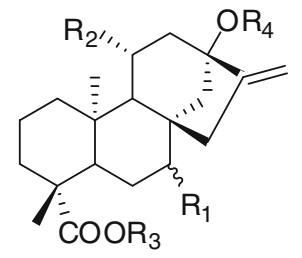

$66 \mathrm{R}_{1}=\mathrm{R}_{2}=\mathrm{H} ; \mathrm{R}_{3}=\mathrm{Glc} ; \mathrm{R}_{4}=\mathrm{Glc}-\mathrm{Glc}$

$67 \mathrm{R}_{1}=\mathrm{R}_{2}=\mathrm{R}_{3}=\mathrm{R}_{4}=\mathrm{H}$

$68 \mathrm{R}_{1}=\mathrm{R}_{2}=\mathrm{R}_{4}=\mathrm{H} ; \mathrm{R}_{3}=\mathrm{Glc}$

$69 \mathrm{R}_{1}=\beta \mathrm{OH} ; \mathrm{R}_{2}=\mathrm{OH} ; \mathrm{R}_{3}=\mathrm{R}_{4}=\mathrm{H}$
Isosteviol (71), an ent-beyerane tetracyclic diterpene obtained from the acid hydrolysis of stevioside (66), possesses several biological activities including the inhibition of D-glucose and D-fructose transport across the cell membrane in isolated perfused rat liver cells. Furthermore, these derivatives displayed potent inhibition of tumor promoters, systemic blood pressure reduction and smooth blood-vessel muscle relaxation, as a cardioprotective effect on rats with heart ischemia-reperfusion injury and reduced plasma

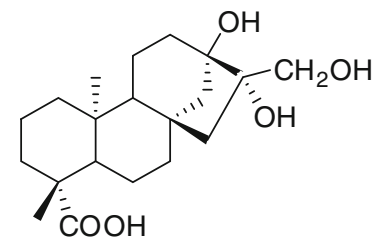

70 
glucose levels in a diabetic animal model (Hsu et al. 2002; Chang et al. 2008).

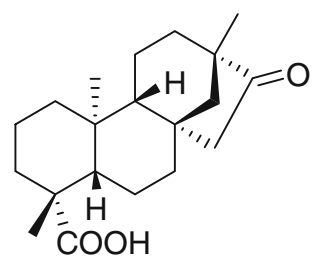

71

Two new oxidized metabolites, $\mathbf{7 2}$ and $\mathbf{7 3}$, were obtained from the biotransformation of $\mathbf{7 1}$ using $M$. recurvatus MR 36 to evaluate the anti-hypertensive activity of these derivatives. In addition, the metabolites were evaluated for anti-hypertensive activity and were found to be less active than the parent compound 71 (Hsu et al. 2002).

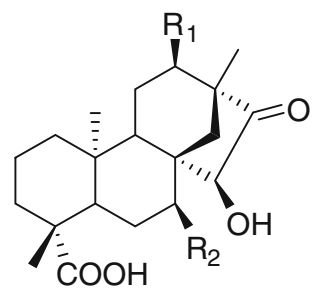

$72 \mathrm{R}_{1}=\mathrm{OH} ; \mathrm{R}_{2}=\mathrm{H}$

$$
73 \mathrm{R}_{1}=\mathrm{H} ; \mathrm{R}_{2}=\mathrm{OH}
$$

Biohydroxylation is a powerful tool for the regioselective and stereoselective introduction of hydroxyl groups at difficult positions on diterpenoid compounds. Thus, as part of an ongoing study to obtain additional hydroxylated compounds for pharmaceutical research, the biocatalytic modification of isosteviol (71) was performed by Chang et al. Both GRE (glucocorticoid response element) and ARE (androgen response element)-mediated luciferase reporter gene assays were used to screen $\mathbf{7 1}$ and the biotransformed products as glucocorticoid and/or androgen agonists. $M$. recuvatus MR 36 was used for mono and dihydroxylation on 71. Derivatives $\mathbf{7 4}$ and $\mathbf{7 5}$ exhibitied a significant effect on ARE-mediated luciferase activity; in particular, $\mathbf{7 5}$ was more active than testosterone (Chang et al. 2008).

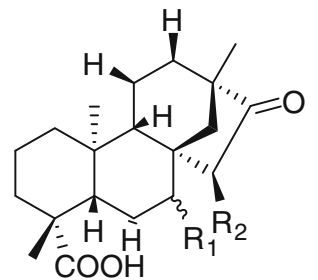

$74 \mathrm{R}_{1}=\mathrm{H} ; \mathrm{R}_{2}=\mathrm{OH}$

$75 \mathrm{R}_{1}=\alpha \mathrm{OH} ; \mathrm{R}_{2}=\mathrm{H}$

Activator protein-1 (AP-1) is recognized as an important modulator in inflammatory diseases such as rheumatoid arthritis, psoriasis and psoriatic arthritis. The inhibition or modulation of AP-1 activation may represent an attractive target for developing novel therapeutic agents for treating inflammatory-mediated conditions. The tetracyclic diterpenoids have a structural similarity to steroids and have been used to develop immunoinflammatory agents. Isosteviol lactone (76), an ent-beyerane tetracyclic diterpenoid, was prepared from isosteviol (71) and biotransformed by $M$. recurvatus $\mathrm{MR} 36$ to bring about structural modifications and to new biological activities. The production, isolation and structural characterization as well as inhibitory effects on AP-1 activation in lipopolysaccharide (LPS)-stimulated macrophages of isosteviol lactone (76) metabolites were reported. All hydroxylated metabolites obtained from the biotransformation of $\mathbf{7 6}$ by $M$. recurvatus MR 36 exhibited significant inhibitory activity on AP-1 activation and 77 was more potent than the reference compound dexamethasone (Chou et al. 2009).

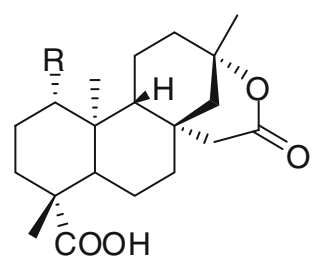

$76 \mathrm{R}=\mathrm{H}$

\section{$77 \mathrm{R}=\mathrm{OH}$}

Fusicoccanes-type diterpenes are significant biosynthetic plant growth regulators. Hypoestenone (78) 


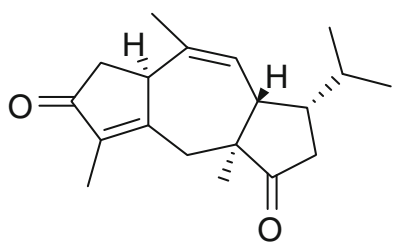

78

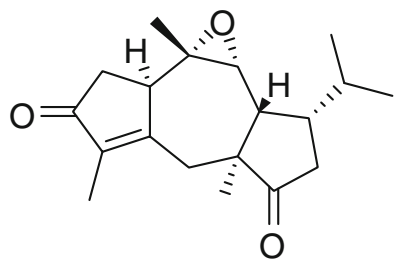

79

Fig. 12 Biotransformation of hypoestenone (78) to 8(9)ג-epoxy-hypoestenone (79) by M. ramannianus ATCC 9628

is a fusicoccane diterpene ketone reported from the aerial parts of Hypoests forskalei (Acanthaceae), and was chosen by El Sayed for a microbial bioconversion study in an attempt to prepare new bioactive analogues. This was the first microbial metabolism study on a member of this significant class of bioactive natural products. M. ramannianus ATCC 9628 was selected for preparative-scale fermentation of $\mathbf{7 8}$, which was converted into five more polar metabolites which were tested for antimicrobial activity against a wide range of microorganisms. Hypoestenone (78) and 8(9) $\alpha$-epoxy-hypoestenone (79) (Fig. 12) exhibited moderate antimicrobial activity against Cryptococcus neoformans (El Sayed 2001).

The leaf and flower cuticular wax of most Nicotiana species contain high amounts of cembranoid diterpenes such as $(1 S, 2 E, 4 R, 6 R, 7 E, 11 E)$ 2,7,11-cembratriene-4,6-diol (80). They possess 14-membered macrocyclic rings substituted by isopropyl residue at $\mathrm{C}-1$ and by three symmetrically arranged methyl groups at positions C-4, C-8, and $\mathrm{C}-12$. Tobacco cembranoids block the expression of the behavioral sensitization to nicotine and inhibit neuronal acetylcholine receptors in rats suggesting a possible use of these compounds for the treatment of nicotine addiction. Tobacco cembranoids also exhibit insecticidal, prostaglandin, plant growth, and fungal spore germination inhibition activity. Cembranoid $\mathbf{8 0}$ from cigarette smoke condensate was reported as an antitumor-promoter ingredient in 1985. Therefore, studies subjected the readily available tobacco cembranoid $\mathbf{8 0}$ to semisynthetic and biotransformation methods in an attempt to further optimize anticancer activity. The biotransformation of $\mathbf{8 0}$ by $M$. ramannianus ATCC 9628 afforded its (10S, 11S)-epoxy analogue as the main metabolite which exhibited less antiproliferative activity than its parent 80 (El Sayed et al. 2008).

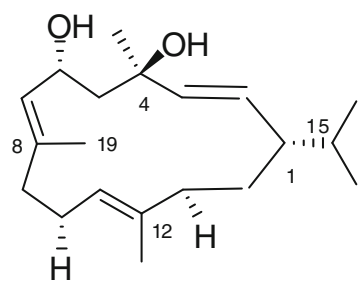

80

In 2011, Baraka et al. isolated the cembranoid $(1 S, 2 E, 4 R, 6 R, 7 E, 11 E)$-2,7,11-cembratriene-4-O-methyl4,6-diol (81) from N. tabacum leaves. Cembranoid 81 exhibited good anti-migratory activity against prostate cancer cell lines and was therefore subjected to microbial transformation by $M$. ramannianus ATCC 9228. The aim of the group was to gain further insight to establish a preliminary structure-activity relationship for the anti-migratory activity of tobacco $(4 R)$-cembranoids through the use of microbial and semisynthetic optimization of 81. M. ramannianus ATCC 9228 afforded four metabolites (82-85) from the biotransformation of $\mathbf{8 2}$. When the anti-migratory activity of cembranoids 82-85 against the highly mestastic PC-3 human prostate cancer cell lines was evaluated, it was discovered that all analogues were generally less active than their parent $\mathbf{8 1}$ (Baraka et al. 2011). 


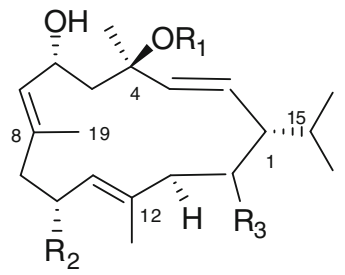

$81 \mathrm{R}_{1}=\mathrm{CH}_{3} ; \mathrm{R}_{2}=\mathrm{H} ; \mathrm{R}_{3}=\mathrm{H}$

$82 \mathrm{R}_{1}=\mathrm{CH}_{3} ; \mathrm{R}_{2}=\mathrm{H} ; \mathrm{R}_{3}=\mathrm{OH}$

$83 \mathrm{R}_{1}=\mathrm{H} ; \mathrm{R}_{2}=\mathrm{OH} ; \mathrm{R}_{3}=\mathrm{H}$

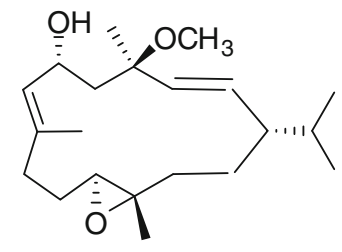

84

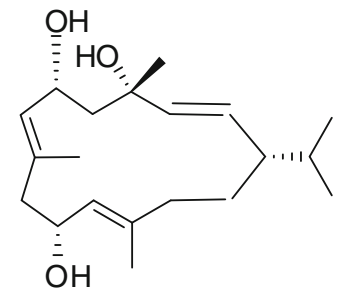

85
The diterpenoid salvinorin A (86) is a natural product with hallucinogenic properties and has become a lead compound in the search for new drugs to combat psychiatric disorders such as schizophrenia, Alzheimer's and other dementias in which $\kappa$-opioid receptors are believed to be involved. The in vivo metabolism of salvinorin A (86) is not fully understood. Although studies with isolated body fluids from primates and humans, previously incubated with 86 revealed salvinorin B (87) as a metabolite, in vivo pharmacokinetic experiments with nonhuman primates failed to detect salvinorin B (87) or any other metabolite in the bloodstream. Prompted by these conflicting observations, Kutrzeba et al. conducted studies of fungal transformation and ex vivo metabolism with salvinorin A (86). Thirty fungal species such as M. plumbeus ATCC 8773, M. ramannianus ATCC 9628 and M. mucedo ATCC 20094 were screened for their ability to metabolize 86. It was observed that salvinorin A (86) undergoes swift hydrolysis of the acetate group at carbon atom $\mathrm{C}-2$ resulting in the formation of the pharmacologically inactive product salvinorin B (87). Ex vivo experiments were also performed using organelle fractions isolated from rat liver and brain. Crude tissue homogenate and individual organelles showed that the primary route of salvinorin A (86) metabolism is hydrolysis to salvinorin B (87). No metabolic transformation of $\mathbf{8 7}$ was observed in these studies (Kutrzeba et al. 2009).

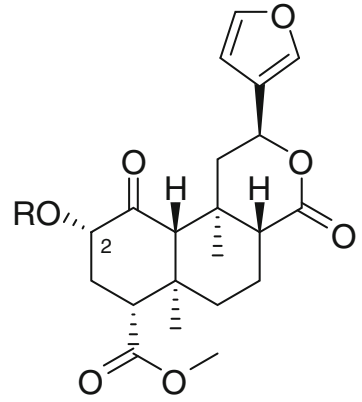

\section{$86 \mathrm{R}=\mathrm{COCH}_{3}$}

\section{$87 \mathrm{R}=\mathrm{H}$}

Podocarpic acid (88) is a naturally occurring diterpene obtained from Podocarpus dacrydioides, P. cupressinus, Dacrydium cupressinum and Falcatifolium taxoides. A number of podocarpic acid (88) analogues have recently been reported as cytokine release inhibitors and, consequently, as leads for the discovery of novel anti-inflammatory drugs. Some analogues have shown antiviral, antitumor, antimicrobial and cytotoxic activities. In this regard, Baraka described the metabolic study of podocarpic acid (88) using M. ramannianus ATCC 9628 to produce new metabolites and to evaluate these metabolites for antioxidant activity. Biocatalysis of podocarpic acid (88) afforded a 2-hydroxy metabolite (89). This derivative displayed a significant level of free radical scavenging activity, greater activity than substrate $\mathbf{8 8}$ (Baraka 2010). 
<smiles>[R3]c1cc2c(c([R])c1O)[C@@]1(C)C[C@@H]([R])C[C@](C)(C(=O)O)[C@]1(CO)CC2</smiles>

$88 \mathrm{R}_{1}=\mathrm{R}_{2}=\mathrm{R}_{3}=\mathrm{H}$

$89 \mathrm{R}_{1}=\alpha \mathrm{OH} ; \mathrm{R}_{2}=\mathrm{R}_{3}=\mathrm{H}$

Several studies have highlighted the antimicrobial activity of diverse natural compounds against cariogenic pathogens. Recently, Severiano et al. have demonstrated that the diterpene ent-pimara-8(14),15dien-19-oic acid (90) is able to inhibit the growth of the main microorganisms responsible for dental cavities with very promising minimal inhibitory concentration (MIC) values. Two pimarane-type diterpenes (91 and 92) were therefore isolated from ent-pimara8(14),15-dien-19-oic acid (90) by means of biotransformation using Mucor rouxii for the purpose of obtaining diterpenes with anticariogenic activity. The structure of these metabolites indicates that isomerization of the endocyclic double bond and oxidation of the chemical structure of $\mathbf{9 0}$ are the main reactions produced by $M$. rouxii (Fig. 13). Evaluation of the anticariogenic activity of these compounds showed low activity (Severiano et al. 2010).

Andrographis paniculata (Burm.f.) Nees is a Chinese herbal medicine used as an anti-inflammatory and antipyretic drug for treatment of fever, cold, laryngitis and diarrhea. Pharmacological research has shown that diterpenoid lactones are the active components of this plant and exhibit a number of bioactive effects. Neoandrographolide (93) is one of the principal components of ent-labdane diterpenoid lactones and has a number of effects: anti-inflammatory, antiviral, anti-radical, hepatoprotective and anti-human immunodeficiency virus (HIV). Wang et al. conducted pharmacological experiments to produce more types of diterpenoid derivatives using different fungi and to identify compounds with better activity than the substrate. Twenty-seven kinds of fungi were screened for the bioconversion of neoandrographolide $(\mathbf{9 3})$ and Mucor spinosus AS 3.2450 proved effective for that purpose. Analysis of the structure of the derivatives obtained showed that many kinds of enzymatic reactions occurred during the biotransformation such as enzymatic hydrolization, hydroxylation, oxidation, glycosylation, epoxidation and elimination. The inhibitory effects of the derivatives on nitric oxide production in lipopolysaccharide-activated macrophages were similar to those exhibited by the substrate. However, the structural features of the labdane skeleton showed that the positions of the hydroxyl and double bond had a significant bearing on activity (Wang et al. 2011).

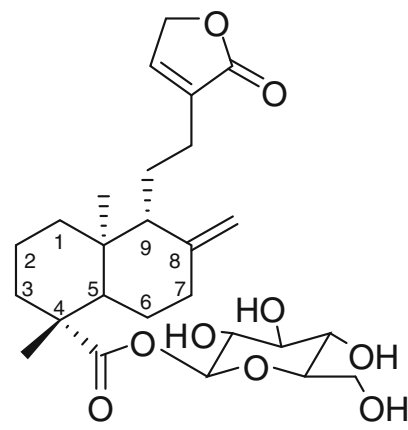

93

\section{Biotransformation of triterpenoids}

Triterpenes are very abundant natural products in the plant kingdom and exhibit huge structural diversity.

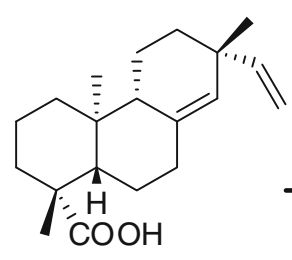

90

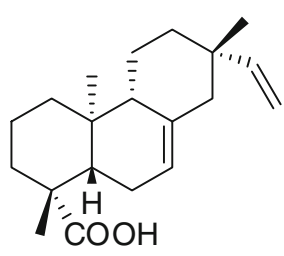

91<smiles>C=C[C@]1(C)CCC2=C(CCC3(C(=O)O)[C@@H](C)CCC[C@@]23C)C1</smiles>

92

Fig. 13 Biotransformation of 90 by $M$. rouxii 
Modification of the triterpenoid structure may produce potentially new and interesting derivatives. Synthetic approaches can be used, but biotransformation processes have been used in order to target positions which are more difficult to functionalize using chemical methods (Carvalho et al. 2010).

Ginsenosides, a special group of triterpenoid saponins, are the major active components in Panax ginseng C. A. Meyer (Araliaceae), and have been reported to exhibit antitumor effects. 20(S)-protopanaxatriol (94) is a ginsenoside aglycone and has been reported to have strong cytotoxic activity against human leukemia cells by inducing DNA fragmentation and apoptosis. In this connection, the biotransformation of 20(S)-protopanaxatriol (94) by $M$. spinosus AS 3.3450 was reported in order to finding more active compounds. The derivative analysis demonstrated that 20(S)-protopanaxatriol (94) was selectively oxidated at position $\mathrm{C}-12$ by M. spinosus AS 3.3450, beyond be hydroxylated at C-15 $\alpha$, C-27 and C-26. The anti-tumoral, cytotoxic effects of the derivatives were evaluated and the results showed that all the metabolites, as well as the substrate, had significant cytotoxic effects on human leukemia cells (Tian et al. 2005).

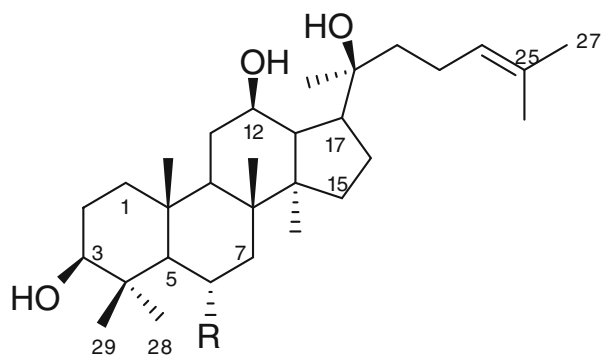

\section{$94 \mathrm{R}=\mathrm{OH}$}

\section{$95 \mathrm{R}=\mathrm{H}$}

In Zhang et al. (2007) reported on the biotransformation of 20(S)-protopanaxatriol (94) by $M$. spinosus AS 3.3450 and various others structurally modified derivatives obtaining enhanced activity and improved bioavailability. The in vitro cytotoxicity of the metabolites on human tumor cell lines was measured, suggesting that the dehydrogenation at
C-12 or hydroxylation at C-28 or C-29 enhanced cytotoxic activity, whereas introduction of a hydroxyl group at C-11 $\beta, \mathrm{C}-15 \alpha, \mathrm{C}-23 \beta, \mathrm{C}-26$ or C-27 reduced the activity of the substrate (Zhang et al. 2007).

M. spinosus AS 3.3450 was also used to biotransform 20(S)-protopanaxadiol (95), which exhibits potent antitumor effects. The $12 \beta$-hydroxyl groups of all the products obtained were selectively dehydrogenated into carbonyl groups while the $3 \beta$-hydroxyl group remained intact. These results indicate that enzymes in the Mucor culture can specifically and efficiently catalyze dehydrogenation of $12 \beta-\mathrm{OH}$. Moreover, the Mucor culture catalyzed the hydroxylation of methane (C-7, C-11, and C-15) and methyl (C-27, C-28, and -29) groups (Li et al. 2009).

The pentacyclic triterpenoid glycyrrhetinic acid (96) is one of the major components of a traditional Chinese medicine, Glycyrrhiza uralensis root. Glycyrrhetinic acid (96) has a number of pharmacological properties: anti-inflammatory, antiviral, antibacterial, antifungal, antiallergic, anticarcinogenic and antiulcerous. The discovery of possibly more active derivatives of 96 will enable the exploitation of more valuable therapeutic agents. To that end, the biotransformation of glycyrrhetinic acid (96) by $M$. polymorphosporus AS 3.3443 was investigated and afforded hydroxylated derivatives at C-24, C-6 $\beta, \mathrm{C}-7 \alpha, \mathrm{C}-7 \beta$ and C- $15 \alpha$, and oxo- and acetyl-derivatives at C-3. Additionally, 24-hydroxyglycyrrhetinic acid (97) obtained in this study was the same as one of the metabolites produced from 96 by rat liver microsomes suggesting some similarities between the microbial and mammalian enzyme systems (Xin et al. 2006). In 2010, other hydroxylated derivatives of glycyrrhetinic acid (96) such as 6 $\beta, 7 \beta$-dihydroxyglycyrrhentic acid and 27-hydroxyglycyrrhentic acid were isolated using M. polymorphosporus (Xin et al. 2010). The major product of microbial transformation of glycyrrhetinic acid (96) by $M$. spinosus AS 3.3450 was identified as a 7 $\beta$-hydroxy derivative. The fact that this fungus hydroxylated glycyrrhetinic acid (96) in a site-specific position is important for the future study of the drug metabolism of this compound in vivo (Ma et al. 2008). 


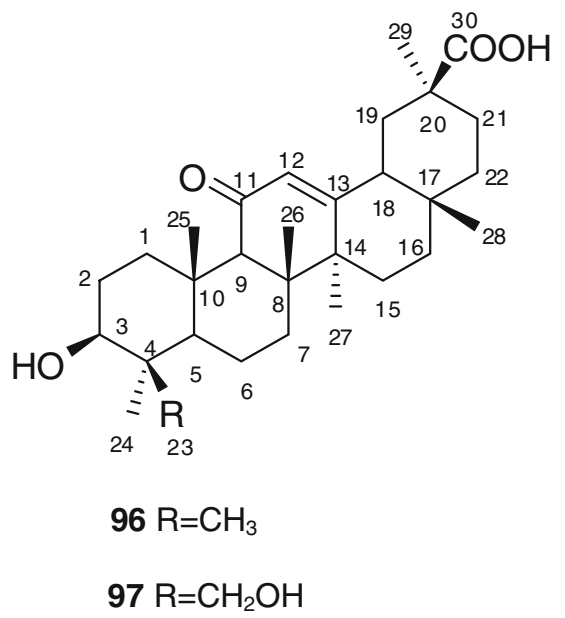

Marine natural products show a diverse array of bioactivity including anticancer, analgesic, antiinflammatory, antiviral, and immunomodulatory activities. Several triterpenoids have been reported from the Red Sea sponge Callyspongia (=Siphonochalina) siphonella. Sipholenol A (98) is one of the major sipholane triterpenoids and exhibits anticancer activities. Thus, Jain et al. isolated sipholenol A (98) from $C$. siphonella and performed the bioconversion of this compound using M. ramannianus ATCC 9628 to generate structurally diverse active derivatives. Additional experiments were conducted to characterize the differential antiproliferative effects of $\mathbf{9 8}$ and its metabolites on several cancer cell lines. Biocatalysis of $\mathbf{9 8}$ afforded several hydroxyl-derivatives which showed milder effects on cell proliferation as compared to the parent compound (Jain et al. 2007).

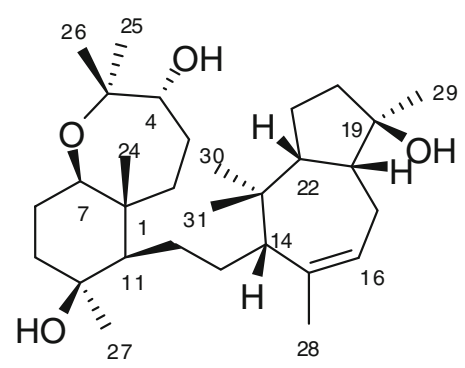

\section{8}

Pentacyclic triterpenes of the lupane type such as lupeol (99) have been reported to exhibit interesting bioactivity including antiviral, anti-inflammatory, and antitumor. The ability of $M$. rouxii NRRL 1894 to biotransform lupeol (99) was reported, affording two metabolites, $\mathbf{1 0 0}$ and 101. The biotransformation processes were influenced by the culture medium and incubation period (Carvalho et al. 2010).

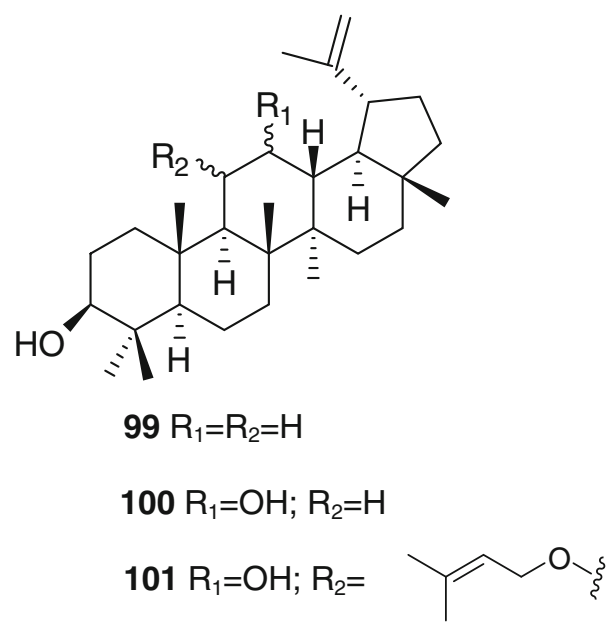

Oleanolic acid (102) is a pentacyclic triterpene widespread in the plant kingdom in the form of free acid or aglycone for triterpenoid saponins. This compound exhibits interesting bioactivity including anti-inflammatory, hepatoprotective, antitumor, antiviral, and antimicrobial activities. Previous studies showed that oleanolic acid (102) and its derivatives display antibacterial activity against oral pathogens. Thus, 102 was biotransformed by $M$. rouxii NRRL 1894 and its derivatives were evaluated against oral pathogens. From among the derivatives obtained from oleanolic acid (102), compound $\mathbf{1 0 3}$ was most active against Porphyromonas gingivalis, which is a primary etiological agent of periodontal disease (Capel et al. 2011).

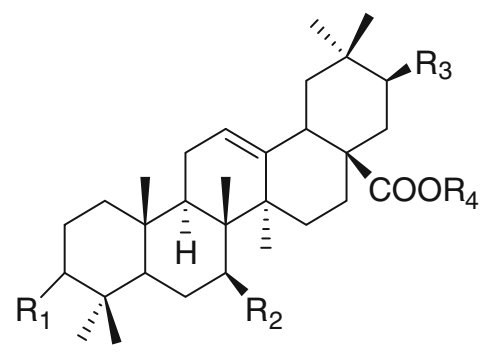

$102 \mathrm{R}_{1}=\mathrm{OH} ; \mathrm{R}_{2}=\mathrm{R}_{3}=\mathrm{R}_{4}=\mathrm{H}$

$103 \mathrm{R}_{1}=\mathrm{O} ; \mathrm{R}_{2}=\mathrm{H} ; \mathrm{R}_{3}=\mathrm{OH} ; \mathrm{R}_{4}=\mathrm{H}$ 
Other important studies on triterpenoid biotransformation by Mucor species have been reported such as the methyl ursolate (104) biotransformation by $M$. plumbeus ATCC 4740 to a single hydroxylated metabolite (Collins et al. 2002) and dioscin (105), a triterpenic saponin which was biotransformed by M. circinelloides MTCC 5263 to diosgenin (106) (Priti et al. 2009).

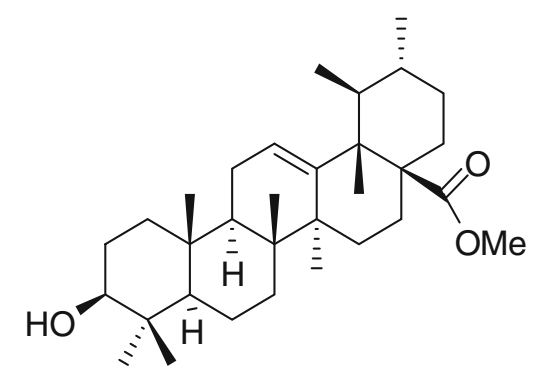

104

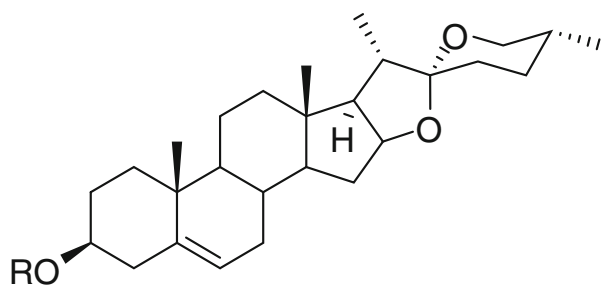

105 R=Glu-Rha-Rha

$$
106 \mathrm{R}=\mathrm{H}
$$

\section{Conclusions}

The high stereospecificity of enzymatic reactions is challenging to organic chemists, because traditional organic chemical reactions often fail to reach the same degree of enantioselectivity. A wide range of reactions are catalyzed by fungi and they are currently considered promising source of new biocatalysts, mainly for chiral reactions and functionalization of deactivated carbons. From results gathered in this review, Mucor species have a considerable enzymatic arsenal that make them attractive tools for the synthesis of compounds which would be difficult to obtain by chemical methods. In the last years, the biotransformations by Mucor species have had a significant importance in the production of high-value products due, principally, to their broad utility for the enantiospecific production of compounds of commercial interest (perfumes, aromas). Thus, the fungal biotransformation of mono- and sesquiterpenoids has offered a very interesting alternative source of natural flavoring.

The biotransformation of diterpenes has proved to be a useful tool in the chemistry of these compounds, specially the stereoselective hydroxylation at deactivated positions. On the other hand, the structural diversification of several taxadienes has yielded derivatives of high pharmaceutical interest and additionally, the biotransformation, by Mucor sp. of xenobiotic substrates has also been used as in vitro models to mimic and predict the mammalian metabolism of biologically active terpenoids.

In the last years, the genomic has transformed the way in which we think about these microorganisms and has greatly influenced the manner in which we probe their chemistry and biology. A number of complete and draft genome sequences are now available and this fact has made Mucor a valuable organism for the study of a number of processes, especially those for industrial applications, pharmacy and medicine.

Finally, it can be concluded that the development of novel biocatalytic methods will continue being a growing area of chemistry, microbiology, and genetic engineering, specially due to the fact that biocatalysts are selective, easy-to-handle, and environmentally friendly. Therefore, the integration of biocatalysis and organic synthesis are sparking the creation of new synthetic strategies and opening up new technological frontiers of both fundamental and practical interest.

\section{References}

Alarcón J, Águila S, Cornejo F et al (2007) Biotransformation of $5 \alpha$-hydroxy-14-eudesm-11-en-3-one by Rhizopus nigricans, Cunninghamella elegans and M. plumbeus. J Mol Catal B Enzym 48:23-27

Aranda G, El-Kortbi MS, Lallemand JV (1991) Microbial transformation of diterpenes: hydroxylation of sclareol, manool and derivatives by $M$. plumbeus. Tetrahedron 47:8339-8350

Arantes SF, Hanson JR, Hitchcock PB (1999) The hydroxylation of the sesquiterpenoid valerianol by M. plumbeus. Phytochemistry 52:1063-1067

Areche C, Loyola LA, Borquez J et al (2008) Microbial transformation of the diterpene mulin-11,13-dien-20-oic acid by M. plumbeus. Magn Reson Chem 46:765-768 
Ata A, Nachtigall JA (2004) Microbial transformations of $\alpha$ santonin. J Biosci 59:209-214

Baraka HN (2010) Microbial transformation of podocarpic acid and evaluation of transformation products for antioxidant activity. Planta Med 76:815-817

Baraka HN, Khanfar MA, Williams JC et al (2011) Bioactive natural, biocatalytic, and semisynthetic tobacco cembranoids. Planta Med 77:467-476

Bartnicki-Garcia S (1968) Control of dimorphism in Mucor by hexoses: inhibition of hyphal morphogenesis. J Bacteriol 96:1586-1594

Boaventura MAD, Oliveira AB, Hanson TJR et al (1995) The biotransformation of methyl ent-15-oxokaur-16-en-19oate by Rhizopus stolonifer and M. plumbeus. Phytochemistry 40:667-1669

Cano A, Ramírez-Apan MT, Delgado G (2011) Biotransformation of sclareolide by filamentous fungi: cytotoxic evaluations of the derivatives. J Braz Chem Soc 22:1177-1182

Capel CS, Souza ACD, Carvalho TC et al (2011) Biotransformation using $M$. rouxii for the production of oleanolic acid derivatives and their antimicrobial activity against oral pathogens. J Ind Microbiol Biotechnol 38:1493-1498

Carvalho TC, Polizeli AM, Turatti ICC et al (2010) Screening of filamentous fungi to identify biocatalysts for lupeol biotransformation. Molecules 15:6140-6151

Chang S, Yang L, Lo C et al (2008) Microbial transformation of isosteviol and bioactivities against the glucocorticoid/ androgen response elements. J Nat Prod 71:87-92

Chen ARM, Ruddock PLD, Lamm AS et al (2005) Stemodane and stemarane diterpenoid hydroxylation by $M$. plumbeus and Whetzelinia sclerotiorum. Phytochemistry 66:1898-1902

Chou B, Yang L, Chang S et al (2009) Fungal transformation of isosteviol lactone and its biological evaluation for inhibiting the AP-1 transcription factor. Phytochemistry 70:759-764

Collins DO, Ruddocka PLD, Grassea JC et al (2002) Microbial transformation of cadina-4,10(15)-dien-3-one, aromadendr-1(10)-en-9-one and methyl ursolate by M. plumbeus ATCC 4740. Phytochemistry 59:479-488

Dai J, Qu R, Zou J et al (2008) Structural diversification of taxanes by whole-cell biotransformation. Tetrahedron 64:8102-8116

El Sayed KA (2001) Microbial transformation of hypoestenone. J Nat Prod 64:373-375

El Sayed KA, Laphookhieo S, Yousaf M et al (2008) Semisynthetic and biotransformation studies of $(1 S, 2 E, 4 S, 6 R, 7 E$, 11E)-2,7,11-cembratriene-4,6-diol. J Nat Prod 71:117-122

Elmarakby SA, El-Feralv FS, Elsohly HN et al (1988) Microbiological transformations of artemisinic acid. Phytochemistry 27:3089-3091

Esmaeili A, Saad N, Shilla S et al (2009) Biotransformation of (-)-menthol by spores of $M$. ramannianus and study of the pathways involved. Herba Polonica 55:51-58

Fraga BM, Guillermo R, Hanson JR et al (1996) Biotransformation of cedrol and reported compounds by M. plumbeus. Pytochemistry 42:1583-1586

Fraga BM, González P, Guillermo R et al (1998) Microbiological transformation of manoyl oxide derivatives by M. plumbeus. J Nat Prod 61:1237-1241

Fraga BM, Hernández MG, González P et al (2001) Biotransformation of the diterpene ribenone by M. plumbeus. Tetrahedron 57:761-770
Fraga BM, Alvarez L, Suárez S (2003) Biotransformation of the diterpenes epicandicandiol and candicandiol by M. plumbeus. J Nat Prod 66:327-331

Fraga BM, Guillermo R, Hernández MG et al (2004) Biotransformation of two stemodane diterpenes by M. plumbeus. Tetrahedron 60:7921-7932

Fraga MB, Alfonso I, Gonzalez-Vallejo V et al (2010) Microbial transformation of two $15 \alpha$-hydroxy-ent-kaur-16-ene diterpenes by M. plumbeus. Tetrahedron 66:227-234

Fraga BM, González-Vallejo V, Guillermo R (2011) On the biotransformation of ent-trachylobane to ent-kaur-11-ene diterpenes. J Nat Prod 74:1985-1989

Furusawa M, Hashimoto T, Noma Y et al (2005) Highly efficient production of nootkatone, the grapefruit aroma from valencene, by biotransformation. Chem Pharm Bull 53:1513-1514

Furusawa M, Hashimoto T, Noma Y et al (2006) Biotransformation of aristolane- and 2,3-secoaromadendrane-type sesquiterpenoids having a 1,1-dimethylcyclopropane ring by Chlorella fusca var. vacuolata, Mucor species, and Aspergillus niger. Chem Pharm Bull 54:861-868

García-Granados A, Martinez A, Parra A et al (2007) Manoyloxide biotransformations with filamentous fungi. Curr Org Chem 11:679-692

Hanson JR, Hitchcock PB, Pibiri I et al (2003) The biotransformation of the diterpenoid rosenonolactone by M. plumbeus. J Chem Res 3:147-149

Hoffmann JJ, Fraga BM (1993) Microbial transformation of diterpenes: hydroxylation of 17-acetoxy-kolavenol acetate by $M$. plumbeus. Phytochemistry 33:827-830

Hollmann M, Razzazi-Fazeli E, Grajewski J et al (2008) Detection of 3-nitropropionic acid and cytotoxicity in M. circinelloides. Mycotoxin Res 24:140-150

Hsu F, Hou C, Yang L et al (2002) Microbial transformations of isosteviol. J Nat Prod 6:273-277

Jain S, Shirode A, Yacoub S et al (2007) Biocatalysis of the anticancer sipholane triterpenoids. Planta Med 73:591-596

Jia N, Chia MX, Lan XX et al (2008) Structural determination of two new sesquiterpenes biotransformed from germacrone by $M$. alternate. Magn Reson Chem 46:178-181

Khalifa SI, Baker JK, Jung M et al (1995) Microbial and mammalian metabolism studies on the semisynthetic antimalarial, deoxoartemininin. Pharm Res 12:1493-1498

Kuban M, Ongen G, Bedir E (2010) Biotransformation of cycloastragenol by Cunninghamella blakesleeana NRRL 1369 resulting in a novel framework. Org Lett 12:4252-4255

Kutrzeba LM, Karamyan VT, Speth RC et al (2009) In vitro studies on metabolism of salvinorin A. Pharm Biol 47: 1078-1084

Li H, Ye M, Guo H et al (2009) Biotransformation of 20(S)-protopanaxadiol by $M$. spinosus. Phytochemistry 70:1416-1420

Ma XC, Wu LJ, Guo DA (2006) Microbial transformation of dehydrocostuslactone by $M$. polymorphosporus. J Asian Nat Prod Res 8:713-718

Ma X, Zheng J, Guo D (2007) Microbial transformation of dehydrocostuslactone and costunolide by $M$. polymorphosporus and Aspergillus candidus. Enzym Microb Technol 40:1013-1019

Ma J, Xin XL, Zhang W et al (2008) Microbial transformation of glycyrrhetinic acid by M. spinosus. Beijing Huagong Daxue Xuebao 35:79-82 
Ma L, Liu X, Liang J et al (2011) Biotransformations of cinnamaldehyde, cinnamic acid and acetophenone with Mucor. World J Microbiol Biotechnol 27:2133-2137

Maatooq GT (2002) Microbial metabolism of partheniol by M. circinelloides. Phytochemistry 59:39-44

Madyastha KM, Thulasiram HV (1999) Transformation of a monoterpene ketone, $(R)-(+)$-pulegone, a potent hepatotoxin, in M. piriformis. J Agric Food Chem 47:1203-1207

Medeiros SF, Avery MA, Avery B et al (2002) Biotransformation of 10-deoxoartemisinin to its $7 \beta$-hydroxy derivative by M. ramannianus. Biotechnol Lett 24:937-941

Miao Z, Wang Y, Yu X et al (2009) A new endophytic taxane production fungus from Taxus chinensis. Appl Biochem Microbiol 45:81-86

Miyazawa M, Nobata M, Hyakumachi M et al (1995) Biotransformation of (+)- and (-)-camphorquinones by fungi. Phytochemistry 39:569-573

Niu J, Ma XC, Xin XL et al (2008) Structural determination of two new sesquiterpenes biotransformed from germacrone by $M$. alternate. Magn Reson Chem 46:178-181

Orabi KY (2001) Microbial epoxidation of the tricyclic sesquiterpene presilphiperfolane angelate ester. J Biosci 56:223-227

Parshikov IA, Miriyala B, Muraleedharan KM et al (2005) Biocatalysis of the antimalarial artemisinin by M. ramannianus strains. Pharm Biol 43:579-582

Priti S, Surrinder K, Chandra TS et al (2009) Biotransformation process for the preparation of diosgenin from dioscin. Indian Patent IN 2008DE00609 A 20090918

Ruiz-Herrera J (1993) Dimorphism in Mucor species. In: Bossche HV, Odds FC, Kerridge D (eds) Dimorphic fungi in biology and medicine. Springer, United States, pp 257-265

Severiano ME, Simao MR, Porto TS et al (2010) Anticariogenic properties of ent-pimarane diterpenes obtained by microbial transformation. Molecules 15:8553-8566

Smith RV, Rosazza JP (1975) Microbial models of mammalian metabolism. J Pharm Sci 64:1737-1759

Souza GC, Anconi CPA, Cornelissen S et al (2009) Selective activity of $M$. plumbeus reductase towards (-)-camphorquinone. J Ind Microbiol Biotechnol 36:1023-1027
Tian Y, Guo H, Han J et al (2005) Microbial transformation of 20(S)-protopanaxatriol by $M$. spinosus. J Nat Prod 68:678-680

Wang Y, Tan T, Tan GK et al (2006) Microbial transformation of the sesquiterpenoid (-)-maalioxide by M. plumbeus. Phytochemistry 67:58-61

Wang Y, Tan T, Tan GK et al (2009) Microbial transformation of the sesquiterpenoid (-)-maaliol by $M$. plumbeus. Tetrahedron 65:5680-5683

Wang Y, Chen L, Zhao F et al (2011) Microbial transformation of neoandrographolide by M. spinosus (AS 3.2450). J Mol Catal B Enzym 68:83-88

Xin X, Liu Y, Ye M et al (2006) Microbial transformation of glycyrrhetinic acid by $M$. polymorphosporus. Planta Med 72:156-161

Xin XL, Yang G, Gou ZP et al (2010) Structural determination of two new triterpenoids biotransformed from glycyrrhetinic acid by M. polymorphosporus. Magn Reson Chem 48:164-167

Yang L, Qu R, Dai J et al (2007a) Specific methylation and epoxidation of sinenxan A by M. genevensis and the multidrug resistant tumor reversal activities of the metabolites. J Mol Catal B Enzym 46:8-13

Yang L, Hsu F, Chang S et al (2007b) Microbial metabolism of steviol and steviol-16a,17-epoxide. Phytochemistry 68: $562-570$

Zhan J, Zhang Y, Guo H et al (2002) Microbial metabolism of artemisinin by Mucor polymorphosporus and Aspergillus niger. J Nat Prod 65:1693-1695

Zhang J, Zhang L, Wang X et al (1998) Microbial transformation of 10-deacetyl-7-epitaxol and $1 \beta$-hydroxybaccatin I by fungi from the inner bark of Taxus yunnanensis. J Nat Prod 61:497-500

Zhang J, Guo H, Tian Y et al (2007) Biotransformation of 20(S)protopanaxatriol by M. spinosus and the cytotoxic structure activity relationships of the transformed products. Phytochemistry 68:2523-2530 